\title{
Correction: The Cochrane HPV vaccine review was incomplete and ignored important evidence of bias
}

Jørgensen L, Gøtzsche PC, Jefferson T. The Cochrane HPV vaccine review was incomplete and ignored important evidence of bias. BMJ Evidence-Based Med 2018;23:165-8. doi:10.1136/ bmjebm-2018-111012

This article (https://ebm.bmj.com/content/23/5/165) has a correction. The changes are clarificatory, and for this reason, the editors have issued a correction and not a retraction. A linked Editor's note provides more background to this decision, and a marked copy is available to view (online supplementary appendix 1) .

The corrections are outlined below.

An additional table outlining the authors' reassessment of 20 studies identified as additionally eligible for the Cochrane HPV vaccine review has been included in the article (online supplementary table 1). Sixteen additional trials were eligible for inclusion in the Cochrane HPV Review (not 20 as stated in the article). Additional data from four trials already included in the Cochrane HPV Review (NCT00929526; NCT00518336; NCT00652938; NCT00578227) are potentially eligible for inclusion.

The number of randomised participants could be assessed for 42 of the 46 trials, and the authors found an additional 25550 females (and possibly up to 30195 for the Cochrane HPV Review's serious adverse events meta-analyses) who are eligible for the Cochrane HPV Review's meta-analyses. In the analysis, the authors did not originally subtract the male participants that were included in three of the studies.

The PATRICIA trial publication only included two thirds (1400/2028) of the serious adverse events listed on ClinicalTrials.Gov". The PATRICIA trial registry reports the total number of women with serious adverse events within each MedDRA preferred term category, ${ }^{1}$ which yields a different total number of women than the total described in the PATRICIA published report and also in the Cochrane HPV Review. ${ }^{2}$ The NCT entry reports the number of women with serious adverse events for each MedDRA term. The final total number of serious adverse events as a proportion of total events remains unknown.

Industry trial funding and other conflicts of interest:

"The Costa Rican Vaccine Trial is a longstanding collaboration between investigators in Costa Rica and NCI. The trial is sponsored and funded by NCI (N01-CP-11005) with support from the NIH Office of Research on Women's Health and conducted in agreement with the Ministry of Health of Costa Rica." The trial publication reports that the "Vaccine was provided for our trial by GSK (GlaxoSmithKline) Biologicals, under a Clinical Trials Agreement with NCI. GSK also provided support for aspects of the trial associated with regulatory submission needs of the company under FDA (Food and Drug Administration) BB-IND 7920. D R Lowy and J T Schiller are named inventors on the US government-owned HPV vaccine patents that are licensed to GSK and Merck, and so are entitled to limited royalties as specified by federal law.”

In the context of FDA regulations, the trial may have been sponsored by GSK, but it is not clear if the trial received any funding from GSK. We consider it is reasonable to accept that GSK provided funding, at least in some kind, since it provided vaccines and support related to the regulatory submission. Therefore, all included trials were funded or sponsored by the HPV vaccine manufacturers.

( Author(s) (or their employer(s)) 2020. No commercial re-use. See rights and permissions. Published by BMJ.

BMJ Evidence-Based Medicine 2020;25:e3. doi:10.1136/bmjebm-2018-111012corr1

Check for updates 